with seizures but normal EEG background at 24 hrs of life had a normal outcome. Background pattern on the a-EEG at onset of seizures or status epilepticus is the best predictor of outcome. (van Rooij LG et al. Pediatrics 2007;120:e354-e363).

H-MRS shows a better correlation with outcome of newborns with HIE compared to conventional MRI. a-EEG has a high sensitivity for alterations in the H-MRS performed in the subacute period after perinatal asphyxia, and both a-EEG and H-MRS have a similar sensitivity and specificity for the prediction of outcome of HIE in term newborn. These a-EEG data obtained at 24 hrs of life may be useful in prediction of subsequent development of seizures in newborns with HIE.

\title{
INFANTILE SPASMS CONSENSUS REPORT
}

An Infantile Spasms Working Group (ISWG) of 14 pediatric neurologists with expertise in IS participated in a 2-day workshop to discuss 1) the current state of IS management, 2) the evidence for efficacy of ACTH and vigabatrin (VGB), approved in the US in 2009, and need for additional alternatives, and 3) to develop protocols as a guide for the diagnostic workup and management of IS. The incidence of IS ranges from 2 to 3.5 per 10,000 live births, and the lifetime prevalence at 10 years of age is lower at 1.5 to 2 per 10,000 children (Trevathan et al, 1999), a result of high mortality. The ISWG discussed the AAP/AAN/CNS consensus statement and practice guideline of 2004, and reviewed the current literature. A consensus was not reached on initial treatment dosage levels, but consensus was strong for the following conclusions:

1) Need for broad clinical evaluation and diagnostic workup, with overnight inpatient 24-hr video-EEG (awake and all stages of sleep, especially non-REM sleep) conducted as soon as possible after identification of spasms. When EEG hypsarhythmia (Gibbs EL \& FA, 1952, preferred spelling) is documented, MRI and other diagnostic tests should be performed and treatment initiated promptly.

2) ACTH and VGB have proven efficacy and are first-line treatments. VGB is first choice for IS comorbid with tuberous sclerosis, and second or third choice with other symptomatic or cryptogenic IS. Duration of therapy, cumulative dose, and daily dose are implicated as risk factors for visual field changes with VGB; periodic ophthalmic evaluations are recommended in addition to baseline, and at 3-6 months after VGB treatment withdrawal.

3) Timely assessment of treatment efficacy ( 2 weeks for ACTH followed by taper; 2 weeks or less following dose titration for VGB). Longer treatment trials are not likely to be effective and may be associated with serious adverse effects.

4) Response to treatment should be "all or none," with complete cessation of spasms and resolution of hypsarrythmia.

(Pellock JM, Hrachovy R, Shinnar S, Nordli DR, et al. Infantile spasms: a US consensus report. Epilepsia Oct 2010;51(10):2175-2189)(Respond: John M Pellock MD, Children's Pavilion, 1001 East Marshall Street, Richmond, VA 23298. E-mail: jpellock@mcvh.vcu.edu).

COMMENT. In a commentary from Finland on the US consensus report (Riikonen R. Epilepsia 2010;51:2215-6), the dangers of prolonged therapy with either ACTH or VGB are emphasized, and the smallest effective doses for 2 weeks are favored. 
Fukuyama Y (Epilepsia 2010;51:2216-7), in a commentary from Japan, prefers the term West syndrome to infantile spasms, and notes differences in treatment protocols in US and Japan. In Japan, the dose of ACTH recommended is smaller and the courses, shorter. Vitamin B6 in large doses is advocated as the first-line treatment for newly diagnosed West syndrome patients. VGB is not available in Japan.

Commentaries from Europe and the UK (Dulac O et al. Epilepsia 2010;51:22189) favor a selective diagnostic and therapeutic approach. Given etiologies for IS determine specific clinical/EEG/imaging patterns. Hypoxic-ischemic encephalopathy, a known cause of WS, has a different course for pre-term and full-term infants. IS following preterm delivery of infants with HIE respond well to treatment, whereas HIErelated spasms following full-term delivery tend to be more severe and refractory. Spasms without hypsarrhythmia, but only focal or multifocal spikes, may suggest focal cortical dysplasia or tuberous sclerosis complex as a cause. These authors are impressed with VGB as first-line therapy, and consider the ketogenic diet no more effective than pyridoxine or conventional AEDs. Parents should be informed of the importance of the EEG in diagnosis, and pediatricians made aware of the early manifestations of WS before the onset of typical spasms. A lack of visual contact or awareness in an infant may signal the onset of WS and the need for wake and sleep EEG, if apparent visual impairment is unexplained by ophthalmologic examination. Early diagnosis and treatment appear to be the key to success in the management of West syndrome.

\section{LONG-TERM OUTCOME OF ENCEPHALOPATHY WITH STATUS EPILEPTICUS DURING SLEEP SYNDROME (ESESS)}

Researchers from the Epilepsy Unit, Helsinki University Central Hospital, Finland, prospectively evaluated the efficacy of antiepileptic drug treatment and longterm (5 years or longer) cognitive outcome in 32 children with ESESS. Epilepsies were atypical rolandic (AR) in 6 children, associated with Landau-Kleffner syndrome (LKS) in 9 , and symptomatic in 17. Prospective treatment with valproate (VPA) combined with ethosuximide (ESM) in 17 children was effective in $3(18 \%)$. Electrical status epilepticus during sleep was abolished in 16 patients. Pre-ESESS cognitive level was regained in a total of $10(31 \%)$ children (4 with AR, 3 with LKS, and 3 with symptomatic epilepsy). The majority had permanent cognitive impairment. Younger age at ESESS diagnosis, lower IQ at diagnosis, and no response to drug treatment were predictive of an unfavorable cognitive outcome. Cognitive outcome depends on treatment response, on EEG and seizures, and on etiology. VPA combined with ESM was the most effective drug treatment. Eight of 16 nonresponders underwent epilepsy surgery. (Liukkonen E, Kantola-Sorsa E, Paetau R, Gaily E, Peltola M, Granstrom M-L. Long-term outcome of 32 children with encephalopathy with status epilepticus during sleep, or ESES syndrome. Epilepsia Oct 2010;51(10):2023-2032). (Respond: Dr Elina Liukkonen, Epilepsy Unit, Helsinki University Central Hospital, PoB 280, 00029 HUS, Finland. E-mail: elina.liukkonen@hus.fi).

COMMENT. SES (or CSWS), the term used for the EEG phenomenon, is defined as spike and wave discharge in $>85 \%$ of non-rapid eye movement (REM) sleep. The encephalopathy ESESS has a poor long-term outcome. Only one third cases regain a 